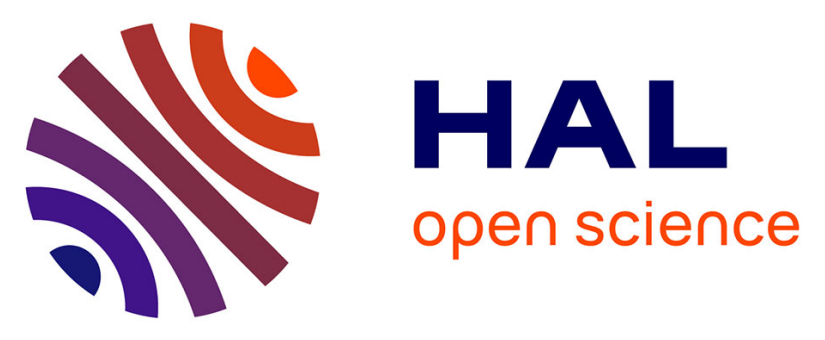

\title{
Embedded Learning for Smart Functional Electrical Stimulation
}

Sebastian Marzetti, Valentin Gies, Valentin Barchasz, Herve Barthelemy, Herve Glotin, Edith Kussener, Remy Vauche

\section{To cite this version:}

Sebastian Marzetti, Valentin Gies, Valentin Barchasz, Herve Barthelemy, Herve Glotin, et al.. Embedded Learning for Smart Functional Electrical Stimulation. 2020 IEEE International Instrumentation and Measurement Technology Conference (I2MTC), May 2020, Dubrovnik, Croatia. pp.1-6, 10.1109/I2MTC43012.2020.9128681 . hal-03386618

\section{HAL Id: hal-03386618 \\ https://hal.science/hal-03386618}

Submitted on 20 Dec 2021

HAL is a multi-disciplinary open access archive for the deposit and dissemination of scientific research documents, whether they are published or not. The documents may come from teaching and research institutions in France or abroad, or from public or private research centers.
L'archive ouverte pluridisciplinaire HAL, est destinée au dépôt et à la diffusion de documents scientifiques de niveau recherche, publiés ou non, émanant des établissements d'enseignement et de recherche français ou étrangers, des laboratoires publics ou privés. 


\title{
Embedded Learning for Smart Functional Electrical Stimulation
}

\author{
Sebastián Marzetti*, Valentin Gies*‡, Valentin Barchasz ${ }^{\ddagger}$, Hervé Barthelemy*, Hervé Glotin ${ }^{\dagger}$, Edith Kussener*§, \\ Remy Vauche* \\ *Aix Marseille Université, Université de Toulon, CNRS, IM2NP - UMR 7334, France \\ ${ }^{\dagger}$ Aix Marseille Université, Université de Toulon, CNRS, LIS - UMR 7020, France \\ $\ddagger$ Université de Toulon, SMIoT, France \\ $\S$ ISEN, Toulon, France
}

\begin{abstract}
This paper presents a smart embedded Functional Electrical Stimulator (FES), able to stimulate a muscle only when a specific movement pattern occurs. This pattern is detected using an inertial measurement unit (IMU) coupled with a feature detector and a neural classifier. Architecture of the FES is first presented, then embedded processing algorithms composed of feature extraction and neural network classification are detailed. Results show that the muscle vibration happening when stimulation is needed can be recognized in more than $90 \%$ of cases using less than $3 \%$ of average embedded processor resources on a $A R M M 4 F$.

Index Terms-Functional Electrical Stimulator, Embedded Learning, Pattern Detection, Embedded system
\end{abstract}

\section{INTRODUCTION}

Functional Electrical Stimulation (FES) is an important medical device for rehabilitating or strengthening patients having injuries in order to restore their walking function, or for preventing muscular atrophy on disabled subjects. Common areas of application are mostly the upper and lower extremities [1].

In this paper, we focus on the design of a smart non invasive FES device, which can be used in medical environments as well as in patient's homes. Stimulation is done by applying an electrical pulse on nerves to shrink targeted muscles and to induce natural limbs movements. Stimulating signal is applied via surface electrode on human skin using a biphasic pulse with null average value. This increases the time before patients get fatigue, allowing the use of the stimulator device for longer time [2].

The stimulating pulse has three key parameters having effects on muscular fatigue: shape, frequency and duty cycle. Shape of the biphasic signals is usually a square wave that improves cells and fiber recruitment compared to other waveforms [3]. Common frequency values are 5 to $50 \mathrm{~Hz}$, with a pulse width of $50 \mu \mathrm{s}$ to $2 \mathrm{~ms}$. It is important to note that electrical stimulation leads to a long or short term muscular fatigue whatever the parameters used are [1], [4]-[7]. This fatigue can even lead to tetanization.

Consequently, there is a strong interest to use FES only when it is really necessary; namely, when muscles are no more able to contract without assistance.

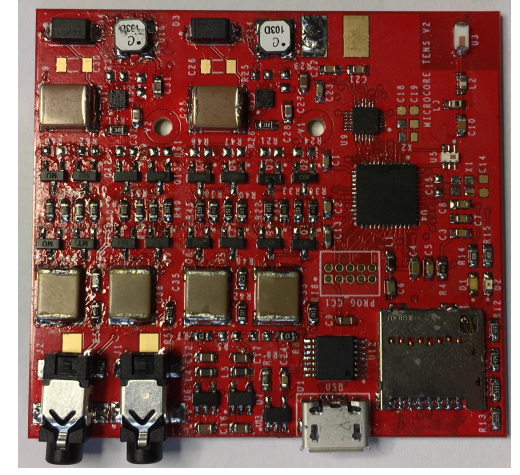

Fig. 1: Picture of the presented smart FES

In our FES, detection of these situations is done using an Inertial Measurement Unit (IMU), coupled with embedded signal processing feature detectors and a neural network for classifying them.

In this paper, we focus on a smart but low cost and low supply voltage FES that can be embedded in a portable Internet of Things (IoT) device. Smart features, such as a detector triggered only when stimulation is necessary, are not present in most of the FES implementations existing in the literature [5], [7]-[10]. A recent work presents an embedded solution (cost around $70 \$$ ) working on a $3 \mathrm{~V}$ power supply, producing $46 \mathrm{~V}$ biphasic pulses [10]. This voltage is too low for a medical use (output voltage up to $120 \mathrm{~V}$ is necessary to be efficient with any type of skin). Another recent work presents an embedded solution working on a $6 \mathrm{~V}$ power supply composed of 2 coin cells $C R 2032$ [7]. Output voltage is limited to $21 \mathrm{~V}$, which is again not useable in medical applications. One more recent work is focused on an electric muscle stimulator (EMS) with an interesting error amplifier closed loop, but its voltage amplification is limited to a factor 20 because of the structure of the boost converter, leading to a maximum voltage of $74 \mathrm{~V}$ on a $3.7 \mathrm{~V}$ lithium cell [9]. This voltage is also too limited for medical applications.

Another work uses fly-back power converters and a push pull current stage which requires double high voltage supply of $+150 \mathrm{~V}$ and $-150 \mathrm{~V}$ [8], which cannot also work on batteries 
and are not relevant for IoT applications.

These solutions have in common several limitations. First, their output voltage amplification is limited. An explanation is that the boost converters used are usually working correctly with duty cycles up to $90 \%$ [11], corresponding to an amplification by a factor 10 , but are not stable at higher amplifications. In our case, we want to amplify the input voltage from $3.7 \mathrm{~V}$ to $120 \mathrm{~V}$ or more. This leads to a boost converter amplification factor of more than 40 . Second, used pulses generators work mainly with integrated solutions limited to voltages lower than $100 \mathrm{~V}$ and are relatively expensive when low-cost applications are targeted (less than $10 \$)$.

For these reasons, a novel smart FES implementation is presented in this paper, which is divided in four parts :

- In section II, architecture and circuits of the smart FES is presented.

- In section III, embedded algorithms and neural classifiers used for smart detection are presented.

- In section IV, the results and a discussion are presented

- In section V, conclusion is presented.

\section{Smart Functional Electrical Stimulator ARCHITECTURE}

Proposed FES architecture is presented in Fig.2. It can be supplied with a single $3.7 \mathrm{~V}$ Li-Ion battery whereas it generates output pulses going up to $120 \mathrm{~V}$, with a bill of materials reduced to less than $20 \$$.

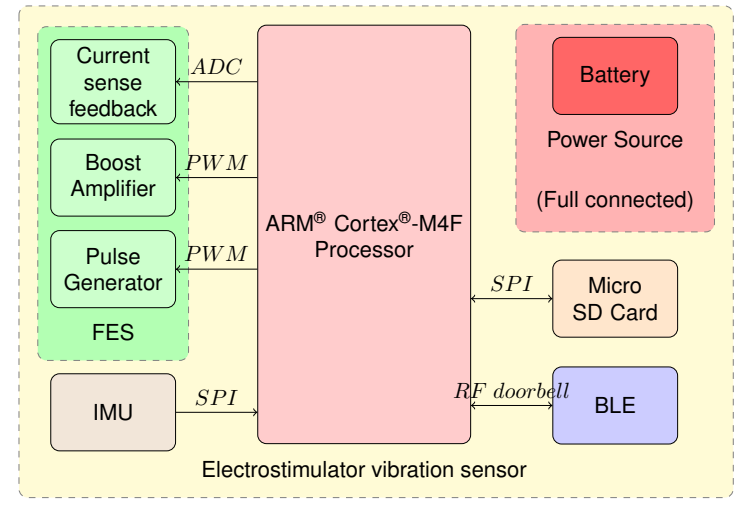

Fig. 2: System block diagram.

The most important parts of this architecture are :

- An electro stimulation circuit: this electrical part is composed of a boost converter amplifying the voltage from $3.7 \mathrm{~V}$ to $120 \mathrm{~V}$, a pulse generator for generating biphasic pulses, and a current sense and feedback for adjusting current applied to the patient.

- An Inertial Measurement unit (IMU) for movement capture.

- A micro-controller for system management and movement processing, including feature extraction and neural network pattern recognition.

All these parts are detailed in the following sub-sections.

\section{A. Electro Stimulation Circuit}

It aims at amplifying voltage and generating controlled current pulses applied to the patient. It is composed of different blocks with specific features.

1) Boost Converter: When operating on a single cell battery in a low power and low cost embedded system, generating a high voltage is complex. Indeed, using a standard boost converter with an inductor as shown in Fig.3 leads to the equation :

$$
V_{\text {out }}=\frac{V_{\text {in }}}{1-D}
$$

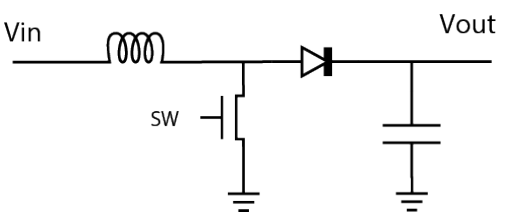

Fig. 3: Standard boost converter

where $D$ is the duty cycle in percent of the period. However, this duty cycle is limited to a maximum value, for example $90 \%$ when using a TPS61170 boost converter [11]. Beyond this value, boost converter is not working properly. From 1, voltage amplification is also limited to 10 using this kind of amplifier, and so output voltage cannot exceed $37 \mathrm{~V}$, which is too low for a versatile FES.

In order to cope with this problem, the standard inductor was replaced with a coupled inductor as shown in Fig. 4.

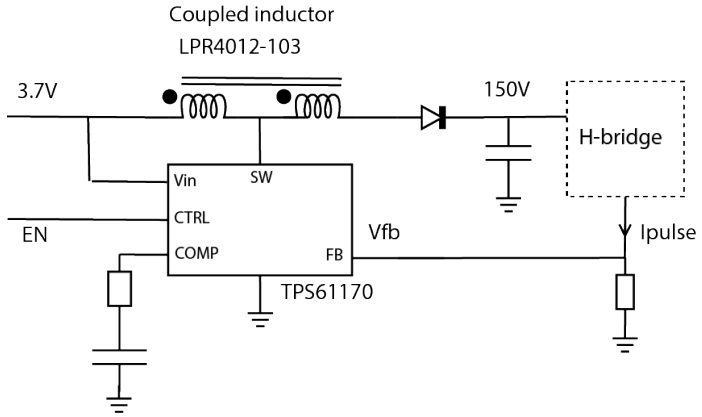

Fig. 4: High voltage boost converter

Using a LPR4012-103 from CoilCraft, the turn ratio of the secondary inductor is 3 , meaning that if a voltage $V_{1}$ is applied on the primary inductor, a voltage $V_{2}=3 V_{1}$ is obtained on the secondary inductor. If both coupled inductors are mounted in series, an amplification by a factor 4 is obtained. Combined with the amplification by a factor 10 obtained with the TPS61170 boost converter, overall amplification is 40 , leading to a maximum output voltage of $144 \mathrm{~V}$ with a $3.7 \mathrm{~V}$ battery in input. This is adequate for our needs and requires only an additional cost in production of $3 \$$.

2) Pulse Generator: Pulse generation is another key feature in FES. Using a high voltage power supply while having a low cost implementation is challenging. In [10], a solution is 
proposed using an integrated $L M D 18200 \mathrm{H}$-bridge, but this solution has two important drawbacks : its input voltage range is limited by the $\mathrm{H}$-bridge to $55 \mathrm{~V}$, and its cost is important. In order to cope with these drawbacks, a discrete components solution has been proposed, relying on standard NMOS and PMOS transistors as shown in Fig. 5.

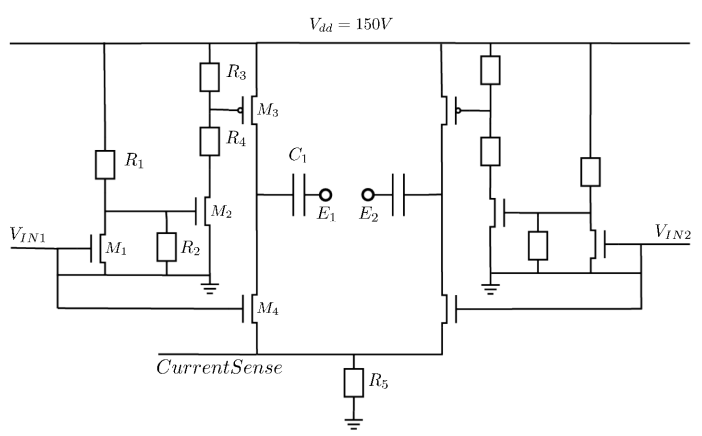

Fig. 5: Functional electrical stimulator

This implementation is composed of 2 symmetrical pulses generators, each one driving as an half bridge composed by a pair of PMOS and NMOS transistors. Transistors must be driven with respect to their maximum gate-source voltage. This absolute value of this voltage is equal to $20 \mathrm{~V}$ for the low cost $Z V P 1320 F$ PMOS transistor used in the design. Maximum gate-source voltage is the same for the $Z V N 3320 F$ NMOS transistor also used in the design. Considering the commands $V_{I N 1}$ and $V_{I N 2}$ are outputs of a micro-controller delivering $3.3 \mathrm{~V}$, the NMOS transistor $M_{1}$ is easy to drive, whereas the PMOS $M_{3}$ is much more complicated, because it requires a potential of more than $130 \mathrm{~V}$ at the gate of the PMOS transistor for being turned on if $V_{d d}=150 \mathrm{~V}$. In order to achieve that, two level shifters are used in input of NMOS transistors $M_{2}$, $M_{4}$ and PMOS transistor $M_{3}$. They work as follows :

1) When $V_{I N 1}=3.3 \mathrm{~V}, M_{1}$ NMOS transistor is turned on, setting the gate voltage of $M_{2}$ NMOS transistor to $0 \mathrm{~V}$. The $M_{2}$ being blocked, gate voltage of the PMOS transistor $M_{3}$ is equal to $V_{d d}$, turning off this transistor whereas the lower NMOS transistor $M_{4}$ is turned on by $V_{I N 1}$, connecting the electrode to the ground.

2) When $V_{I N 1}=0 V, M_{1}$ NMOS transistor is turned off, setting the gate voltage of $M_{2}$ NMOS transistor to $19 \mathrm{~V}$, through the voltage divider composed of $R_{1}=100 \mathrm{k} \Omega$ and $R_{2}=15 k \Omega$. Consequently, transistor $M_{2}$ is turned on, connecting $R_{4}$ to the ground. Gate voltage of $M_{3}$ PMOS transistor is then equal to $0.87 V_{d d}=131 \mathrm{~V}$ due to the voltage divider (level shifter) composed of $R_{4}=$ $100 k \Omega$ and $R_{3}=15 k \Omega$ and this transistor is turned on connecting the electrode to $V_{d d}$.

Pulse generator is fully functional as shown in Fig. 6 in the case where boost output voltage is equal to $V_{d d}=50 \mathrm{~V}$. Using low cost $Z V P 1320 F$ and $Z V N 3320 F$ transistors and the boost converter described in subsection II-A1, turn on and turn off times of pulses are equal to less than $2 \mu s$ with a boost output voltage equal to $150 \mathrm{~V}$. This allows to generate

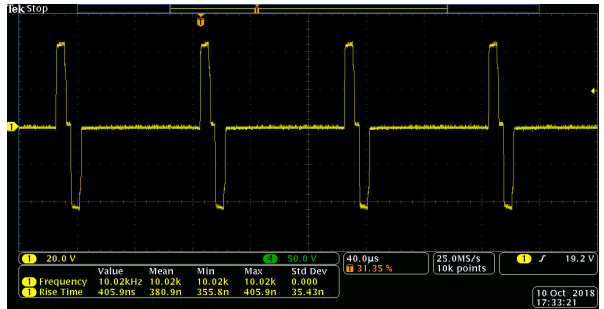

Fig. 6: FES biphasic pulses (in yellow).

biphasic pulses with an extremely precise pulse width control and at a maximum operational frequency limited to $100 \mathrm{kHz}$ as shown in yellow in Fig. 7. It is interesting to notice that output voltage of the boost converter is lowering at very high frequencies, but that is not a problem because skin impedance is lower [12], [13]. In addition, both electrodes are connected to ground when no signal is applied, this is safer than some others implementations where both electrodes are connected to $150 \mathrm{~V}$.

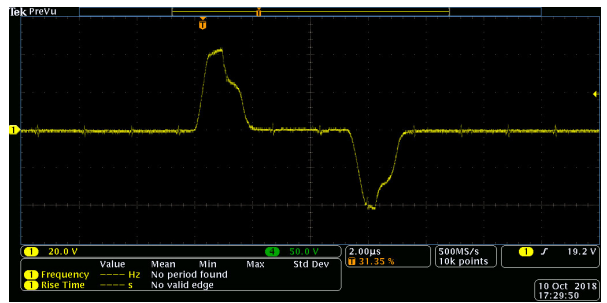

Fig. 7: FES high frequency pulses (in yellow)

Components cost of this portion of circuit is approximately $1.3 \$$ in production, which is really cheap considering the wide range of pulses able to be generated.

3) Current Sense and Feedback: As shown in introduction, body load impedance is sensitive to many parameters and can vary from $10 \mathrm{k} \Omega . \mathrm{cm}^{-2}$ to $1 \mathrm{M} \Omega . \mathrm{cm}^{-2}$ as shown in [12], [13]. For this reason, current in electrodes has to be controlled by a feedback system in order to adjust its value precisely.

A voltage feedback is included in most integrated boost converters in the market. In the TPS61170, a feedback pin $F B$ is used for regulating $V_{F B}$ voltage to a defined value equal to $1.229 \mathrm{~V}$. Inserting a low value resistor $R$ between ground and NMOS transistors of the full-bridge allows to monitor the current $I$ in the electrodes. If this resistor is tied to $F B$, the feedback loop will ensure that the resistor voltage will be equal to $1.229 \mathrm{~V}$, and that the current in the electrodes will be equal to $I=\frac{1.229}{R}$. In the case $R=12.29 \Omega$, current in the electrodes will be fixed to $0.1 \mathrm{~A}$, the maximum value needed. Of course, this current value will be obtained only if the corresponding $V_{d d}$ value doesn't exceed the maximum value that can be produced by the boost converter $(150 \mathrm{~V})$.

Controlling current in the electrode is important, but it has also to be adjustable in order to let the user choose the strength of the FES. In the TPS61170, reference voltage $V_{F B}$ can be adjusted by using a PWM control on the CTRL pin. In this 
case, reference voltage $V_{F B}=$ Duty $\times 1.229 \mathrm{~V}$ where Duty is the duty cycle of the $P W M$ in percent.

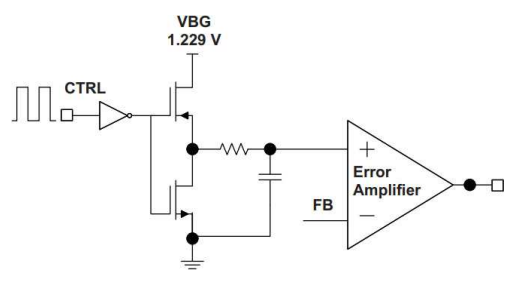

Fig. 8: Boost converter feedback control in TPS91170

This solution relies on a low pass filter as shown in Fig. 8, creating a delay on the feedback reference voltage adjustment. However, this delay is not a problem even at a high pulse repetition frequency with very short pulses.

\section{B. Inertial Measurement Unit}

Assistance of the FES is necessary when the muscle is unable to create a movement by itself. In this case, the muscle often tries to contract without success, leading to a vibration. This pattern can be detected for activating the FES in order to stimulate the muscle.

Capturing moves is done using a low power 9-Axis MEMS Motion Tracking device (TDK ICM-20948), using only its 3 -axis accelerometer with programmable sensitivity of $\pm 2 g$, $\pm 4 \mathrm{~g}, \pm 8 \mathrm{~g}$, or $\pm 16 \mathrm{~g}$, at a maximum sample rate of $1.125[\mathrm{kHz}]$.

Considering muscles vibration base frequency is around $5 \mathrm{~Hz}$, IMU is used at a 100sps (samples per second). Accelerometer only will be used in order to reduce power consumption. Chosen sensitivity value is $\pm 8 g$.

\section{Embedded processor and system management}

A $48 \mathrm{MHz}$ ARM $^{\circledR}$ Cortex $^{\circledR}$-M4F Processor is used for system management, movement processing and pattern detection. Movement data from the 3-axis accelerometer is grabbed using a SPI interface.

The system is powered by a rechargeable battery, charged using the USB connection. A micro SD data storage has been added, enabling to record data used for offline machine learning. Micro SD operations are performed using SPI. USB can be used for real-time monitoring of the system.

ARM $^{\circledR}$ Cortex $^{\circledR}-\mathrm{M} 4 \mathrm{~F}$ processor communicates through a hardware interface called the RF doorbell with a second processor, an $\mathrm{ARM}^{\circledR}{ }^{\circledR}$ Cortex $^{\circledR}-\mathrm{M} 0$ in charge of handling the Bluetooth Low Energy RF protocol used for connecting the smart FES to a smartphone.

\section{EMBEDDED SIGNAL PROCESSING AND NEURAL PATTERN RECOGNITION}

Even though this device can be used for any part of the body, our FES is used for rehabilitation after elbow and shoulder injuries. Our goal is to switch on the FES only when the muscle is unable to create a movement by itself. As explained before, this situation leads to several successive unsuccessful contractions of the muscle, generating a vibration. This pattern has to be detected and classified for activating the FES.

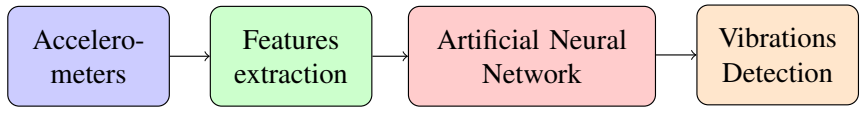

Fig. 9: Feature extraction and pattern recognition chain

Embedded signal processing operations are presented in Fig. 9 and are described in the following subsections.

\section{A. Movement capture}

As a first step, movement is measured using the IMU accelerometer. Fig.10 shows the signal during muscle vibration, and during recovery.

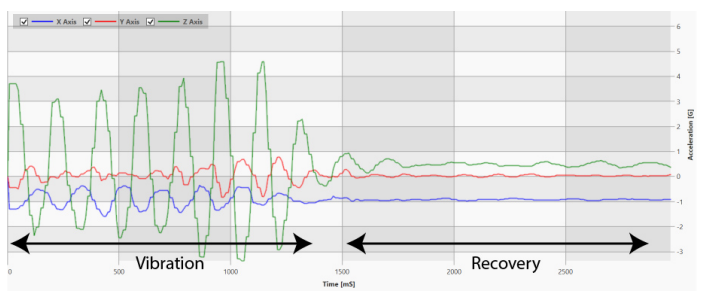

Fig. 10: 3D acceleration during muscle vibration and recovery.

\section{B. Features extraction}

Pattern recognition using neural networks requires high level features extraction from the raw signal [14]. These features have to be as relevant as possible for characterizing the signal.

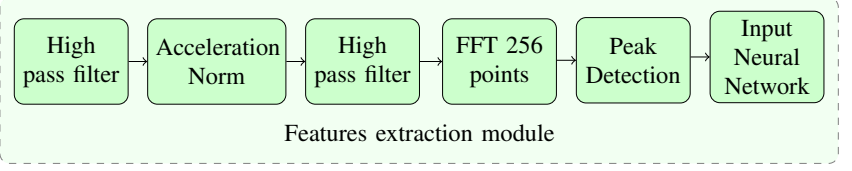

Fig. 11: Features extraction module blocks diagram.

Features extraction is composed of the following succession of operations as described in Fig. 11 :

- removal of the gravity using a $0.5 \mathrm{~Hz}$ high pass filter on acceleration signals $A x, A y$ and $A z$.

- computation of the acceleration magnitude:

- centering of the acceleration magnitude (Fig.12) using a $0.5 \mathrm{~Hz}$ high-pass filter.

- computation of frequency spectrum (Fig.13) using a Fast Fourier Transform (FFT) on 256 points.

- detection of FFT peak : amplitude and frequency of the highest 3 peaks of the FFT spectrum are used as inputs of the neural network, sorting them by amplitude in decreasing sense.

\section{Neural network pattern recognition}

1) Neural Network Structure: Artificial neural network is presented in Figure 14. It is a standard three layers neural network, the first layer (Input Layer) is composed by six neurons (magnitude and frequency of the 3 most important 


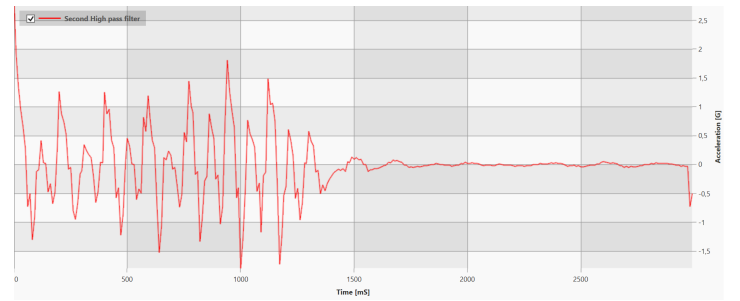

Fig. 12: Centered acceleration magnitude during muscle vibration and recovery.

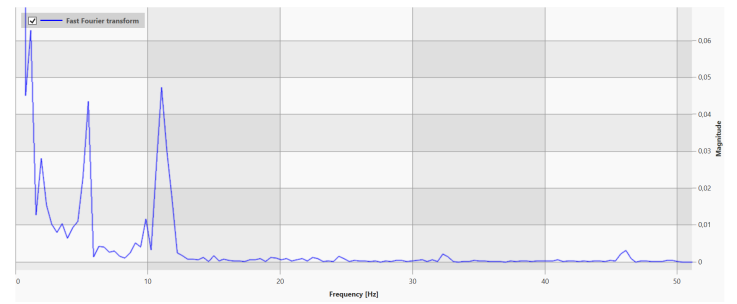

Fig. 13: FFT spectrum of the acceleration magnitude during muscle vibration.

FFT peaks), then second one (Hidden Layer) with forty neurons and the last one (Output layer) is composed by one neuron, telling if the processed movement is classified as relevant vibrations or not. Neural network equations are:

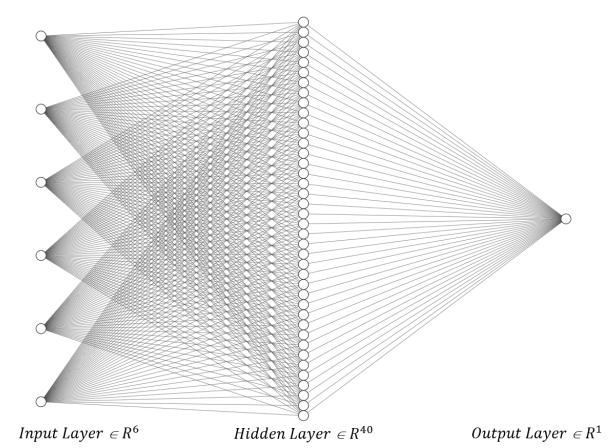

Fig. 14: Artificial neural network for vibrations detection.

$$
z^{[0]}=W^{[0]} a^{[0]}+b^{[0]}
$$

where $z^{[0]}$ is an array of forty elements, $W^{[0]}$ the weights matrix of forty rows and six columns, $b^{[0]}$ the bias array of forty elements and $a^{[0]}$ is the six rows input array with the frequencies and magnitudes.

$$
a^{[1]}=g\left(z^{[0]}\right)
$$

where $g$ is the activation function chosen for the neural network (sigmoid), and $a^{[1]}$ is the output of the second layer with forty elements.

$$
z^{[1]}=W^{[1]} a^{[1]}+b^{[1]}
$$

where $z^{[1]}$ is an array of one element, $W^{[1]}$ the weights matrix of one rows and forty columns and $b^{[1]}$ the bias array of one element.

$$
a^{[2]}=g\left(z^{[1]}\right)
$$

The neural network output is $a^{[2]}$ and it represents the probabilities of a vibration move detection.

2) Training: Training of the pattern recognition neural network in supervised learning has been done off-line in C\#. The data collection involved 10 people ( 3 females and 7 males between 20 and 40 years) and is composed of 290 samples. The data set is divided into a training set of 120 samples, 60 with muscle vibrations and 60 without vibration, and a test set containing 125 samples for no vibration moves and 45 samples for vibration moves. No vibration moves test set is bigger than vibration moves test set because there are many kinds of movement corresponding to a situation without vibration. Learning and testing all these situations is necessary for avoiding false positive detections since it would activate FES module when it is not necessary.

Learnt weights and bias of the network have been saved and transmitted to the micro-controller for embedded real time feature extraction and neural classification.

\section{RESULTS AND DISCUSSION}

\section{A. Classification results}

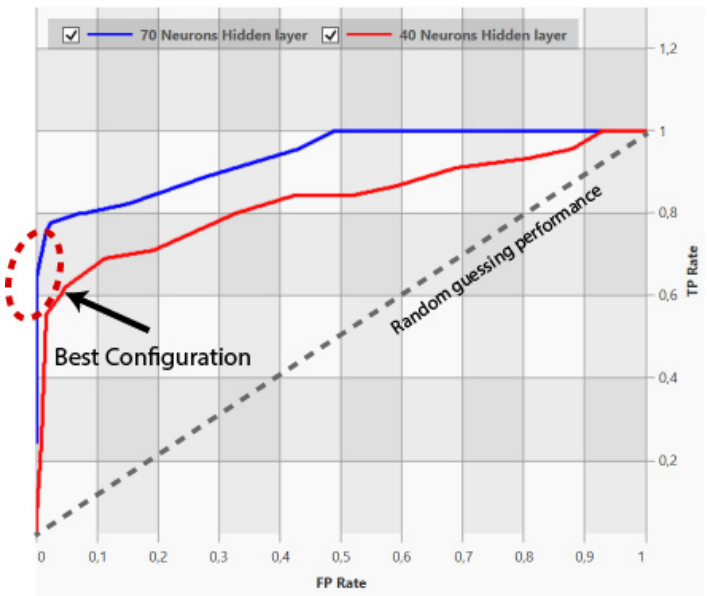

Fig. 15: ANN ROC curves.

For evaluating the performance of the neural network, Receiver Operating Characteristic (ROC) curve and average precision (AP) are used as proposed in [15]. ROC curve is presented in Fig. 15. which shows a comparison between the ANN presented and the same ANN having 70 neurons in the hidden layer instead of 40 . AP of the ANN is presented in Table I. It allows a second comparison for both ANN (40 and 70 neurones in the hidden layer).

\section{B. Computation time}

Embedded processing time for these detection are the following ones: 
TABLE I: ANN Average precision.

Neurons in hidden layer Average Precision

\begin{tabular}{|c|c|}
\hline 40 & 70 \\
\hline 0.739 & 0.886 \\
\hline
\end{tabular}

- Feature extraction:

Fast Fourier Transform: $1.774 m s$.

Peak Detection: $175 \mu \mathrm{s}$.

- Pattern recognition:

Neural Network Computation:

Hidden layer of 40 neurons: $234 \mu \mathrm{s}$.

Hidden layer of 70 neurons: $398 \mu \mathrm{s}$.

At a $100 \mathrm{~Hz}$ accelerometer frequency, detecting the presence of vibration every $100 \mathrm{~ms}$ using a 256 samples window is largely enough. Detection is triggered by a microcontroler internal timer. This leads to a computation time of $23.5 \mathrm{~ms}$ every second using a 70 neurons internal layer, leading to a $2.35 \%$ average usage of the embedded processor for this task.

\section{Discussion}

As shown in the classification results, using a 70 neurons hidden layer on the learning network is better than using 40 neurons, with a very small impact on the overall computation time. Increasing the number of neurons in the hidden layer has been tested, but doesn't improve a lot classification results. Moreover, it can lead to an overlearning if the training set is not large enough.

An optimal situation for our use is to avoid false positives leading to activate FES when it is not necessary. As shown in Fig.15, this can be achieved using a 70 neurons network with a classification threshold equal to 0.7 . In this case, results are the following ones :

- No vibration moves : 125 true detections and 0 classification errors.

- Vibration moves : 29 true detections and 16 classification errors.

Precision of vibration move detection is $T P_{\text {Rate }}=64 \%$, precision of non-vibration move detection is $F P_{\text {Rate }}=0 \%$. This leads to an overall accuracy equal to $90.6 \%$. Moreover, it is important to notice that there are no false positive detection of non-vibration moves : that means the FES is not triggered if it is not necessary.

Many different features have been tested for this work. Our conclusion is that the choice of the features extracted is essential for a good pattern recognition and a high classification rate.

\section{CONClusion}

In this paper, a novel approach for a smart embedded functional electrical stimulation is proposed. In order to reduce muscle fatigue, FES is triggered only when the muscle is not strong enough to make a movement. In this situation, resulting vibrations are detected using an inertial measurement unit coupled with a feature extraction and a neural classifier. Detection accuracy is $90.6 \%$ and requires only $2.35 \%$ of the average embedded processor usage, with no classification errors in the case of non-vibrating moves.

This novel FES can be used for Internet Of Things (IoT) application, such as wearable devices thanks to it low cost (about $10 \$$ ), and its reduced size and power consumption. Moreover, it can be operated on a single $3.7 \mathrm{~V} \mathrm{Li-Ion} \mathrm{cell}$ used very often in IoT applications.

\section{ACKNOWLEDGMENT}

The authors would like to thank the reviewers for their insightful comments and advices, Pôle INPS of Toulon University and the Embedded Electronics Technology Platform of Toulon University named SMIoT (Scientific Microsystems for Internet of Things) [16] for its support in the achievement of this smart FES.

\section{REFERENCES}

[1] P. H. Peckham and J. S. Knutson, "Functional electrical stimulation for neuromuscular applications," Annual Review of Biomedical Engineering, vol. 7, pp. 327-360, 2005.

[2] S. C. Huerta, M. Tarulli, A. Prodic, M. R. Popovic, and P. W. Lehn, "A universal functional electrical stimulator based on merged flybacksc circuit," in 15th International Power Electronics and Motion Control Conference (EPE-PEMC - ECCE), September 2012.

[3] D. M. Durand, "Electric stimulation of excitable tissue," The Biomedical Engineering Handbook, pp. 1-23, 2000.

[4] C. A. Phillips, "Functional electrical stimulation and lower extremity bracing for ambulation exercise of the spinal cord injured individual: A medically prescribed system," Physical Therapy, vol. 69, no. 10, pp. 56-63, October 1989.

[5] S. Simcox, G. Davis, A. Barriskill, J. Middleton, I. Bruinsma, M. Duncan, and R. Smith, "A portable, 8-channel transcutaneous stimulator for paraplegic muscle training and mobility," Journal of Rehabilitation Research and Development, vol. 41, no. 1, pp. 41-52, 2004.

[6] F. Brunetti, . Garay, J. C. Moreno, and J. L. Pons, "Enhancing functional electrical stimulation for emerging rehabilitation robotics in the framework of hyper project," in 2011 IEEE International Conference on Rehabilitation Robotics, June 2011, pp. 1-6.

[7] A. Tamtrakarn, "A portable switching bi-phasic stimulator with levelshifting inverter for functional electrical stimulation operating under a $6 \mathrm{v}$ supply voltage," in TENCON 2015 - 2015 IEEE Region 10 Conference, Nov 2015, pp. 1-4.

[8] M. Ilic, D. Vasiljevic, and D. B. Popovic, "A programmable electronic stimulator for fes systems," IEEE Transactions on Rehabilitation Engineering, vol. 2, no. 4, pp. 234-239, Dec 1994.

[9] M. J. McNulty and P. Fogarty, "Design of a highly efficient circuit for electrical muscle stimulation," in 2006 IEEE Biomedical Circuits and Systems Conference, Nov 2006, pp. 202-205.

[10] A. M. Stewart, C. G. Pretty, and X. Chen, "Design and testing of a novel, low-cost, low-voltage, functional electrical stimulator," in 2016 12th IEEE/ASME International Conference on Mechatronic and Embedded Systems and Applications (MESA), Aug 2016, pp. 1-6.

[11] TPS61170 1.2-A High-Voltage Boost Converter in 2-mm x 2-mm2 QFN Package, Texas Instruments, 11 2007, rev. Dec. 2014.

[12] J. G. Webster, "Skin impedance," Journal of Electrical Bio Impedance, vol. 5, p. 1, 2014

[13] J. Rosell, J. Colominas, P. Riu, R. Pallas-Areny, and J. G. Webster, "Skin impedance from $1 \mathrm{hz}$ to $1 \mathrm{mhz}$," IEEE Transactions on Biomedical Engineering, vol. 35, no. 8, pp. 649-651, Aug 1988.

[14] M. Verhelst and B. Moons, "Embedded deep neural network processing: Algorithmic and processor techniques bring deep learning to iot and edge devices," IEEE Solid-State Circuits Magazine, vol. 9, no. 4, pp. 55-65, Fall 2017.

[15] A. K. Nandi and H. Ahmed, Classification Algorithm Validation. IEEE, 2019, pp. 307-319. [Online]. Available: https://ieeexplore.ieee.org/document/8958927

[16] SMIOT, "Smiot: Scientific microsystems for the internet of things," http://www.smiot.fr, 2019. [Online]. Available: http://www.smiot.fr 